\title{
Problemidentifikation und Motivation
}

In der Literatur gibt es kein ganzheitliches Vorgehensmodell als Handreichung für Praktiker bei der Implementierung von Exoskeletten in intralogistischen Prozessen (vgl. Kap. 2). Dies kann zu einem höheren Risiko von Fehlentscheidungen, vermeidbaren Kosten und einer übermäßig langen Dauer von Einführungsprojekten führen.

Darüber hinaus ist es für Praktiker wichtig im Detail zu verstehen, wie Investitionen in Exoskelette zu Produktivitäts- und Qualitätsverbesserungen und zur Verringerung von Erkrankungen des Bewegungsapparats der Arbeitnehmer beitragen.

Ein Vorgehensmodell in Form eines standardisierten Prozesses strukturiert die Gesamtaufgabe, so dass sich der Fortschritt des Implementierungsprojekts verfolgen und dokumentieren lässt. Darüber hinaus fördert ein Vorgehensmodell das gemeinsame Verständnis des Einführungsprozesses und die funktionsübergreifende Zusammenarbeit zwischen den beteiligten Abteilungen. 